\title{
The Efficiency Performance on Handover's Scanning Process of IEEE802.16m
}

\author{
Ardian Ulvan and Robert Bestak \\ Czech Technical University in Prague, Faculty of Electrical Engineering, \\ Technicka 2, Prague, 16627, Czech Republic \\ \{ulvana1, bestar1\} afel.cvut.cz
}

\begin{abstract}
This paper investigates the efficiency of scanning process on handover procedure in IEEE $802.16 \mathrm{~m}$. The performance evaluation is based on current running standard of $802.16 \mathrm{e}$ since $.16 \mathrm{~m}$ is still under development. The evaluation is done at both parts of handover procedure, i.e. network topology acquisition and handover process. An efficient scanning process is proposed to reduce the scanning time and obtained a minimal handover interruption time.
\end{abstract}

Keywords: WiMAX network, handover, MAC efficiency.

\section{Introduction}

The IEEE 802.16m amends the IEEE802.16 WirelessMAN-OFDMA specification to provide an advanced air interface for operation in licensed frequency bands. It includes enhancements and extensions to the IEEE STD 802.16e to meet the cellular layer requirements of IMT-Advanced next generation mobile networks conducted by International Telecommunications Union - Radio Communications Sector (ITU-R) [1].

The handover is an integral part of all mobile wireless systems. Continuous connection during user movement among cells is allowed due to handover procedure, but on the other hand, the handover brings a significant increase of Medium Access Control (MAC) overhead and also causes an increase in delay of packet delivery to the destination user.

The handover WiMAX is introduced in the latest version of IEEE802.16e [2]. Based on the emerging $802.16 \mathrm{j}$ proposals, it is very probable that in the next versions of WiMAX recommendations (IEEE 802.16j, and IEEE $802.16 \mathrm{~m}$ ) will be defined the same types of handovers. Moreover, the general principles (with regards to the requirements of new standards) of handover will be adopted from 802.16e.

The handover issues on IEEE802.16 have been carried out in several literatures. The fast handover in IEEE802.16e is analyzed in [3]. The single neighbor BS scanning, fast ranging and pre-registration have been proposed to reduce the handover time. To decrease hard handover, the authors in [4] propose handover modification that makes possible to receive downlink data just after synchronization with downlink channel of the target Base Station (BS). This is achieved by introducing a new MAC management message - Fast DL_MAP_IE message, which describes the fast downlink access definition in system's information element. This message is used for 
transmission of emergent packet (packet with payload of delay sensitive services) by target BS to mobile station (MS). In [5], the collision of connection identifiers (CIDs) in the target BS is solved by employing transport CID mapping scheme that increases the handover performance. The authors also introduce Passport handover to decrease the hard handover delay.

The aim of this paper is to analyse scanning time during the network topology acquisition stage of handover procedure and to determine handover interruption time that occurs during the handover procedure. We consider several scan types and several handover strategies in our analysis to obtain the most efficient scanning time and the lowest interruption time.

The remaining of the paper is organized as follows. The description of handover procedure based on IEEE802.16e standard, the analysis of inefficient aspect of handover, scanning time analysis and handover interruption time in IEEE802.16m's system profile are described in Section 2. The result analyses by numerical calculations are given in Section 3. Section 4 concludes our work and highlights our future work.

\section{Handover Procedure}

\subsection{IEEE802.16e Handover}

According to [2], the handover procedure can be divided in two stages (Figure 1). Stage that is executed before handover, called Network Topology Acquisition, contains network topology advertisement and MS scanning. In this stage, the Mobile Station (MS) investigates and collects information about neighborhood base stations of its Serving BS. During the scanning phase, the MS seeks a suitable handover to the target BS or Relay Station (RS) that are suitable to be added to the Diversity Set. The Diversity Set is a list of the BSs/RSs, which are involved in the handover procedure in case of Macro Diversity Handover (MDHO) or Fast Base Station Switching (FBSS).

The scanning is realized in the "scanning intervals" which interleave the normal operation of MS. Once the scanning phase is completed, the MS sends results to its serving BS. The reported results can be delivered by two report types. The first one is "event trigger report", in which MS sends reports based on a defined trigger, such as carrier-to-interference-and-noise ration (CINR), receive signal strength indicator (RSSI), relative delay, or round trip delay (RTD). In this type of report, the measurement report is sent to the Serving BS after each measurement case. In the second type of report, "periodic report", the MS sends reports periodically.

The results of scanning are used in the next stage of handover procedure called Handover Process. The first step is cell reselection. In this step, possible target BS is selected based on signal quality and offered QoS. Then, handover decision and initiation process can be initialized if all conditions and requirements for handover are met follow. The first step of handover process is ended by performing the synchronization to the new Target BS. However, before the synchronization is done the connection(s) to the serving BS have to be closed first.

As soon as the downlink synchronization is done, the MS can start the next step of handover: network re-entry procedure. The network re-entry consists of three substeps 
i.e. ranging, re-authorization and re-registration. In the ranging process the MS obtains information about uplink channel via Uplink MAP (UL-MAP) and Uplink Channel Descriptor (UCD) messages. The Ranging is followed by authorization and registration of MS to the target BS. After successful authorization and registration to the target BS, the MS can start normal operation.

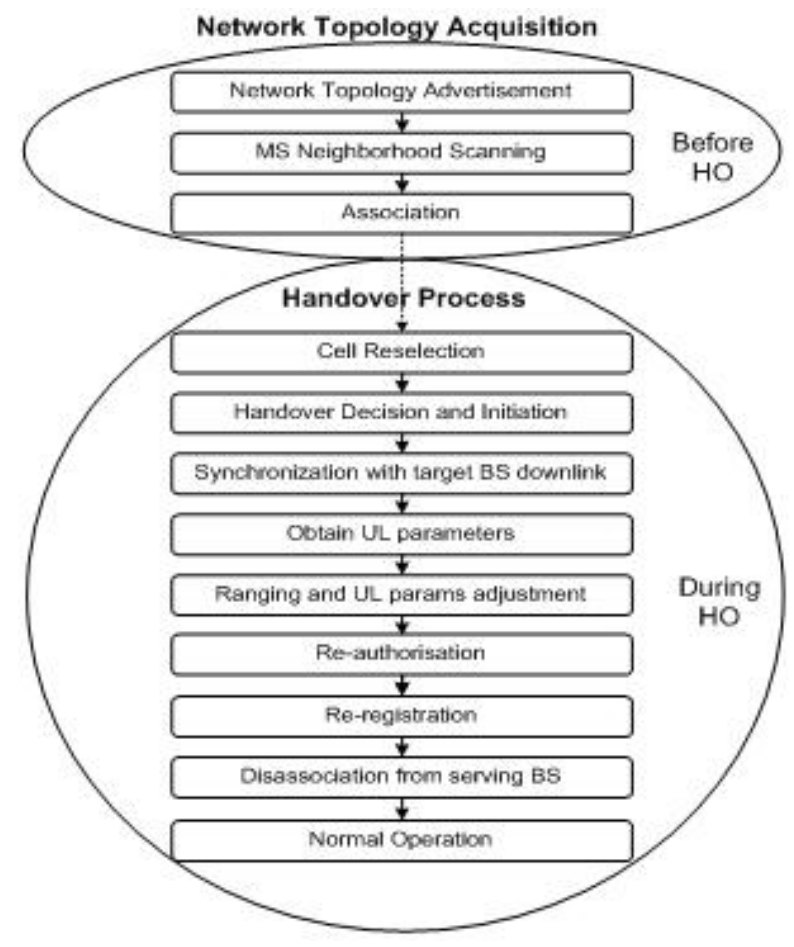

Fig. 1. Two stages of Handover Procedure based on IEEE802.16e

\subsection{MAC Management Overhead in Handover}

The total handover overhead is given by the summarization of length of all handover MAC management messages exchanged during the handover procedure.

Generally, the total handover overhead is affected by following parameters:

- Frequency of handovers (number of handovers per time interval)

- Sequence of exchanged messages

- $\quad$ Length and structure of MAC messages

Frequency of handovers

The number of handovers per a time interval depends on numbers of MSs and $\mathrm{BSs} / \mathrm{RSs}$, speed of MSs, the trajectory of MSs and the setting of cells' boundary. The 
number of MSs and BSs/RSs in a network and speed of MSs are random and cannot be changed or influenced without impact on users QoS. In contrast, the boundary of cells (or range of BS' areas) can be effected by network parameters setting (threshold levels, relative or absolute thresholds, hard handover threshold hysteresis, etc).

\section{Sequence of exchanged messages during handover}

The sequence of management messages is different (but analogical) for different types of handovers. Additionally, the message sequence can even be different for the same types of handover; e.g. in case of different conditions or handover requirements. Besides, the message sequence depends on the initiator of handover procedure (BS or MS). The MAC management message sequence can be modified by network parameter setting up such as periodicity of MS scanning of neighborhood BSs/RSs or scanning results reporting.

\section{Length and structure of MAC messages}

The length of most MAC management messages vary with respect to handover conditions, or types and requirements [2]. The length of messages is affected by proper setting up of handover parameters (BS cell range, thresholds, etc.). For example, the length of messages during scanning phase depends on the number of recommended BSs to scan.

\subsection{Minimization of MAC Management Overhead}

\subsubsection{Reducing the Scanning Time}

In network topology acquisition stage, in fact, there is only one BS that can be selected as target BS for handover. In addition, the result obtained from the scanning process may become invalid because of the changing of neighbour BS's channel quality. Consequently, if the scan or association process occupies too many resources, the throughput significantly decreases. Furthermore, the standard does not clearly state the scanning time. If the scanning is not done with proper timing, channel condition of neighbour BSs may be changed. That would make the scanning process results useless. Figure 2 shows the exchanges of MAC management message in the handover procedure.

These two parts of handover procedure, fulfill up by the MAC layer, contribute to the overhead in $802.16 \mathrm{~m}$. Fortunately, there are several schemes that can be implemented to cope with these issues. The main wastes of resources are represented by redundant scanning and association processes of neighbour BSs. To resolved the issues we propose the single BS scanning scheme, target BS fast ranging and the preregistration mechanism.

Single BS scanning scheme can be achieved by exploiting the target BS estimation algorithm, in which a MS only scans or associates to the neighbour BS with the best CINR. In the mean time, target BS uses a Fast_Ranging_IE to grant a dedicated uplink ranging opportunity to MS in its broadcasting UL-MAP message. So, the MS does not need to do contention-based ranging. Finally, the pre-registration is deployed, in which target BS obtains the service flow and authentication information of this MS through backbone network before handover. 


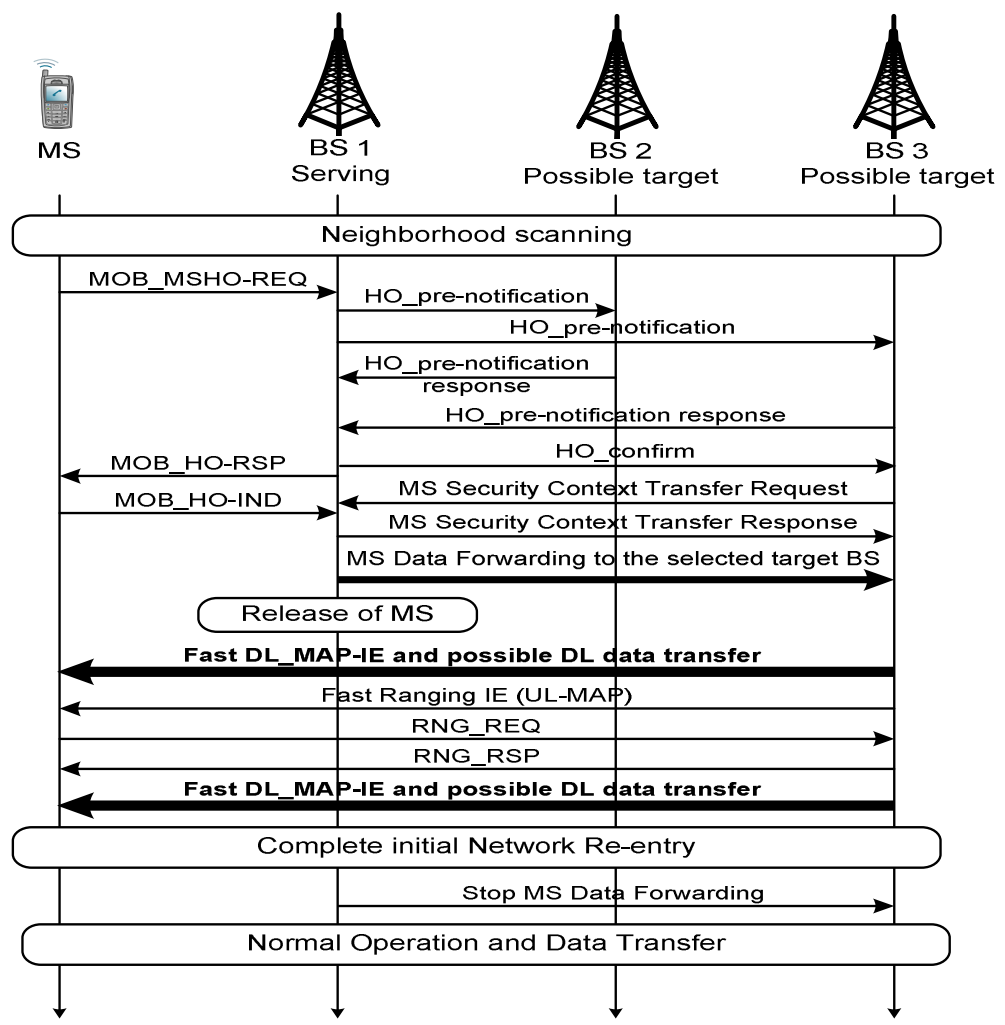

Fig. 2. MAC Management Message exchanges within the handover

To analyze the performance of efficient scanning scheme, we consider four types of scanning. The first type is denoted as $\boldsymbol{S c n}_{\mathbf{1}}$ and it is defined as scanning process of BSs by MS without association. In this scanning type, the MS is only requested to obtain downlink synchronization with the target BS in order to get information about quality of target BS's physical channel. The scanning time can be calculated as:

$$
S c n_{-1}=n \times T_{\text {Sync }}
$$

where $\boldsymbol{n}$ denotes as the number of neighbor BSs that need to be scanned and $\boldsymbol{T}_{\text {Sync }}$ is the average time required for downlink synchronization.

The second type is $\boldsymbol{S c n}_{2}$ and it is defined as scanning process of BSs by MS without coordination. A MS requires the downlink synchronization as well as the execution of contention resolution-based ranging. The $\mathrm{Scn}_{2}$ can be calculated as:

$$
S c n_{-2}=n \times\left(T_{\text {Sync }}+T_{\text {Cont_res }}\right)
$$

where $\boldsymbol{T}_{\text {Cont_res }}$ is defined as the average time required for contention resolution-based ranging (see equation 5 for calculation). 
The third type of scanning is denoted as $\boldsymbol{S c n}_{3}$ and it is defined as scanning process of BS by MS with coordination. A MS requires the downlink synchronization and the execution of fast ranging process. The scanning time can be calculated as:

$$
S c n_{-3}=n \times\left(T_{\text {Sync }}+T_{\text {Rng }}\right)
$$

where $\boldsymbol{T}_{\boldsymbol{R} \boldsymbol{n}}$ is the average time required for fast ranging (see equation 6 for calculation).

Finaly, the fourth type of scanning is denoted as $\boldsymbol{S c n}_{\mathbf{4}}$ and it is defined as scanning process of BS by MS with network assisted association reporting mode. The scanning process is similar to $\boldsymbol{S c n}_{3}$. The difference is that the target BS does not send RNG-RSP message directly to the MS. The RNG-RSP message is firstly sent to the serving BS through backbone network. Then, the serving BS packs all RNG-RSP messages from scanned neighbor BSs into one MOB_ASC-REPORT message and sends it to the MS either during interleaving scanning intervals or normal operation time. Thus, the average time required for fast ranging is assumed to be a half of that for a contention-based ranging. The scanning time for this type of scanning can be calculated as:

$$
S c n_{-4}=n \times\left(T_{\text {Sync }}+\left[\frac{1}{2} \times T_{\text {Rng }}\right]\right)
$$

More details concerning different type of scanning processes can be found in reference [3].

\subsubsection{Performance Analysis of Scanning Time}

In this section we analyze the performance of scanning process. Based on system description document, we assumed a $20 \mathrm{~ms}$ super frame of IEEE $802.16 \mathrm{~m}$ is divided into 4 equally-size frames, where the frame length is assumed to be $5 \mathrm{~ms}$ [8]. The ratio of cell load $\left(\boldsymbol{R}_{\text {Load }}\right)$ is assumed to be $0 \leq R_{\text {Load }} \leq 100 \%$. The $\boldsymbol{T}_{\text {Cont_res }}$ depends on the CINR and ratio of cell load. According to [9] the $\boldsymbol{T}_{\text {Cont_res }}$ is set to $75 \mathrm{~ms}$ and $150 \mathrm{~ms}$ when the ratio of cell load is $0 \%$ and $50 \%$ respectively. Since the ranging collision probability is higher as ratio of cell load increases, then $\boldsymbol{T}_{\text {Cont_res }}$ can be approximated as:

$$
T_{\text {Cont_res }}=75+\left(\left[150 \times R_{\text {Load }}\right] \times 2\right)
$$

The average time required for fast ranging $\left(\boldsymbol{T}_{\boldsymbol{R} n g}\right)$ is also assumed to be directly proportional to the ratio of cell load. The $\boldsymbol{T}_{\boldsymbol{R} n \boldsymbol{g}}$ is set to $25 \mathrm{~ms}$ and $50 \mathrm{~ms}$ when the ration of cell load is $0 \%$ and $50 \%$ respectively [9]. It can be calculated as:

$$
T_{\text {Rng }}=25+\left(\left[50 \times R_{\text {Load }}\right] \times 2\right)
$$

The average time required for authorization $\left(\boldsymbol{T}_{\text {Auth }}\right)$ is relatively longer than the other times since it is the time needed by target BS to obtain the authorization information of MS from the authorization server. $\boldsymbol{T}_{\text {Auth }}$ is assumed to be $150 \mathrm{~ms}$ [3]. Finally, the average time required for registration $\left(\boldsymbol{T}_{\boldsymbol{R} e g}\right)$ is assumed to be 2 frames. The considered values of delays in our paper are summarized in Table 1. 
Table 1. Time paramaters in IEEE802.16m scanning process

\begin{tabular}{ccc}
\hline Time required & Notation & Typical value \\
\hline Synchronization & $\boldsymbol{T}_{\text {Sync }}$ & 2 frames \\
Contention resolution-based ranging & $\boldsymbol{T}_{\text {Cont_res }}$ & hundredth of ms \\
Fast ranging & $\boldsymbol{T}_{\boldsymbol{R} \text { g }}$ & tenth of ms \\
Authorization & $\boldsymbol{T}_{\text {Auth }}$ & $150 \mathrm{~ms}$ \\
Registration & $\boldsymbol{T}_{\boldsymbol{R} \text { eg }}$ & 2 frames \\
\hline
\end{tabular}

The results of scanning time performance with respect to cell load and number of neighbour BSs are depicted in Figure 3 and Figure 4. The discussion of the results can be found on section 3 .

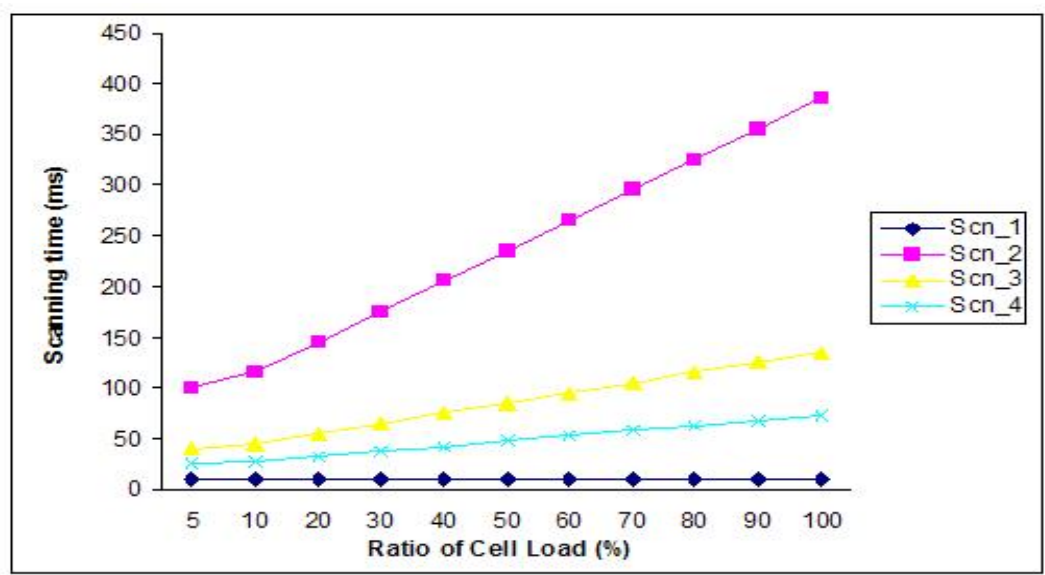

Fig. 3. The scanning performance in various ratio of cell loads

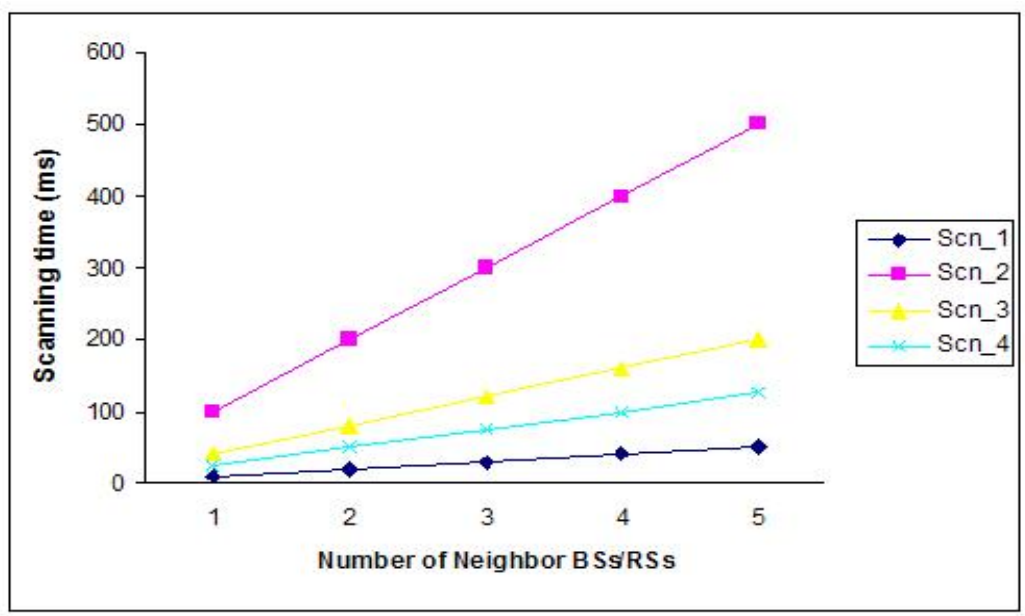

Fig. 4. The scanning performance in various numbers of BS/RS 


\subsubsection{Handover Interruption Time}

The handover interruption time in $802.16 \mathrm{e}$ systems is caused by switching of MS form the serving BS to the target BS The same description is assumed for $802.16 \mathrm{~m}$. The system profile of $802.16 \mathrm{~m}$ also designed the maximum interruption time for handover i.e. $30 \mathrm{~ms}$ for intra-frequency and $100 \mathrm{~ms}$ for inter-frequency [6].

When the MS crosses a boarder of cells between the serving BS and target BS, the connection with the serving BS is closed. After that, a new connection with the target BS is established. Notice that after closing the connection and before setting up the new one, the MS has no connection to the network for a short time. During the interruption, packets must be routed from the serving BS to the target BS. After establishing of the connection between the MS and the target BS, packets are again sent to MS. The packets are delayed due to re-connection (network re-entry) of MS to the target BS [7].

The total interruption time during the handover process depends on the deployed handover strategy. The standard specifies four handover strategies (Table 2, [2]).

Table 2. Handover strategies

\begin{tabular}{cl}
\hline Handover strategy & \multicolumn{1}{c}{ Description } \\
\hline HO_1 & Handover with contention-based ranging \\
HO_2 & Handover with fast ranging \\
HO_3 & Handover with contention-based ranging and pre- \\
HO_4 & registration \\
\hline
\end{tabular}

In case of $\boldsymbol{H O} \_\mathbf{1}$, prior receiving HO-IND message with handover start or service release indicator, serving BS does not inform the target BS to provide dedicated ranging opportunity for MS. During the network re-entry, if collision occurs, MS executes random backoff algorithm and obtain a ranging opportunity through contention. Furthermore, the target BS does not obtain the MS's registration information such as authorization or service flow information from backbone network. Hence, the handover delay of HO_1 can be calculated as:

$$
D_{H_{-} 1}=T_{\text {Sync }}+T_{\text {Cont_res }_{-}}+T_{\text {Auth }}+T_{\text {Re } g}
$$

The $\boldsymbol{H O} \_2$ includes the fast ranging phase, ,but the target BS does not obtain the MS's registration information from the backbone network. Therefore, the MS has to execute a re-authorization and re-registration process. The $\mathrm{HO}_{-} 2$ handover delay can be calculated as:

$$
D_{H_{-} 2}=T_{\text {Sync }}+T_{\text {Rng }}+T_{\text {Auth }}+T_{\operatorname{Re} g}
$$

In case of $H_{0} \mathbf{3}_{3}$, the contention-based ranging and pre-registration schemes are adopted. The target BS obtains the MS's registration information from the backbone network.. Despite the target BS knows the MS's information, it requires to send REQRSP message including CID updating information to the MS. Therefore the average 
time for pre-registration is assumed to be a half of the average time of whole registration process. The handover delay of $\mathrm{HO}_{-} 3$ is given as:

$$
D_{H_{-} 3}=T_{\text {Sync }}+T_{\text {Cont_res }}+\frac{T_{\operatorname{Re} g}}{2}
$$

The last type of handover, $\boldsymbol{H O} \_4$, adopts the fast ranging and pre-registration schemes. The target BS provides a dedicated ranging opportunity to MS and obtaining the service flow and authorization information of the MS from backbone network. The target BS also requires to send REQ-RSP message including CID update information the MS. Thus, the average time required for registration is assumed to be half of complete registration. The handover delay of $\mathbf{H O}-\mathbf{4}$ can be calculated as:

$$
D_{H_{-} 4}=T_{\text {Sync }}+T_{\text {Rng }}+\frac{T_{\operatorname{Re} g}}{2}
$$

The results of handover interruption time of four handover strategies are shown in Figure 5. The results are discussed in section 3.

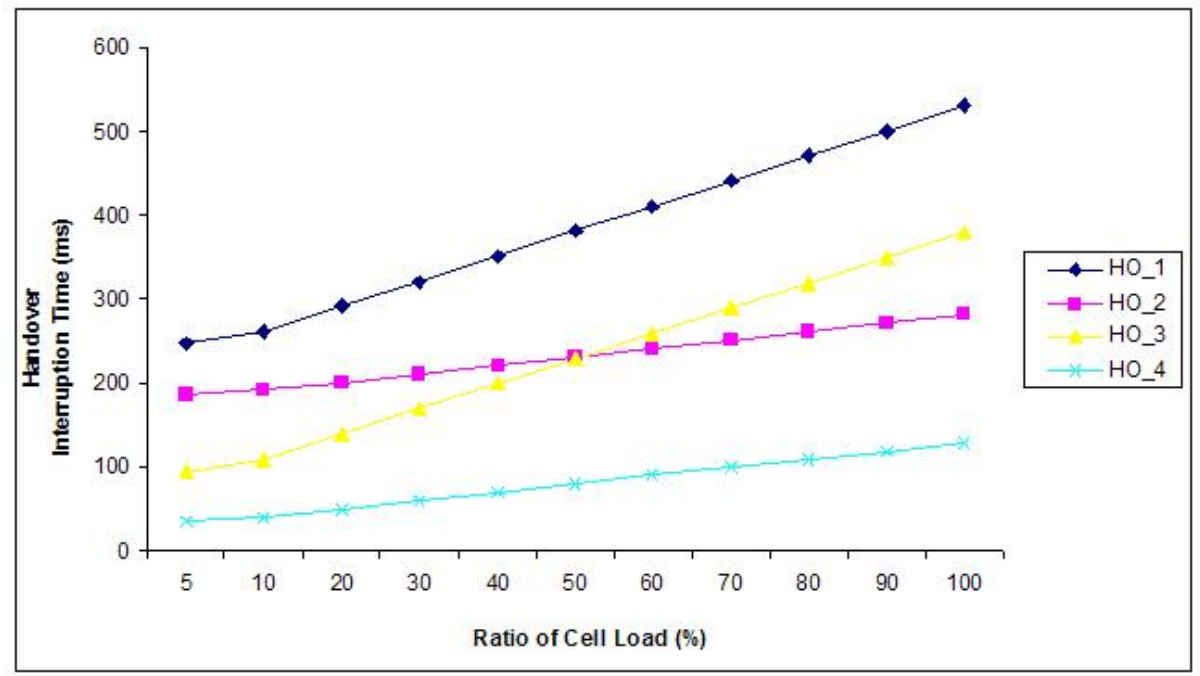

Fig. 5. Handover interruption time in various handover strategies

\section{Analysis of Efficiency}

Based on presented results in previous section, analysis of efficiency of scanning process and handover interruption time is discussed.

Figure 3 shows that the ratio of cell load has significant impact on the scanning time for some of scanning types. In case of the scanning without association $\left(\mathbf{S c n}_{\mathbf{l}}\right)$ the scanning time remains constant for what different cell load increases. This is due 
to the fact that a MS only needs to synchronize with the target BS to learn the quality its physical channel. For other three scanning mechanisms, the scanning time increases as the cell load increases.

From Figure 4, it can be observed that the number of neighbour BSs affects the duration of scanning time. For the ratio of cell load of $5 \%$, and 5 neighbor BSs, the increase of scanning time is almost linear to the increase of number of neighbour BSs. The scanning without association $\left(\boldsymbol{S c n}_{\mathbf{l}}\right)$ also has a better performance than the ones in all cases.

Figure 5 indicates that the performance of handover interruption time depends on several conditions such as the cell load and frame duration. Handover with fast ranging and pre-registration $\left(\boldsymbol{H O} \_4\right)$ seems to be the only handover strategy that can fulfilled the IEEE802.16m's requirements since it can reach the interruption time around $34 \mathrm{~ms}$.

Impact of different frame duration on the scanning time is shown in Figure 6. As it can be observed, the scanning time linearly increase as the number of frame growing. Again, the scanning type $\boldsymbol{S c n}_{\mathbf{I}}$ gives better results since it has the lowest scanning time (10 ms).

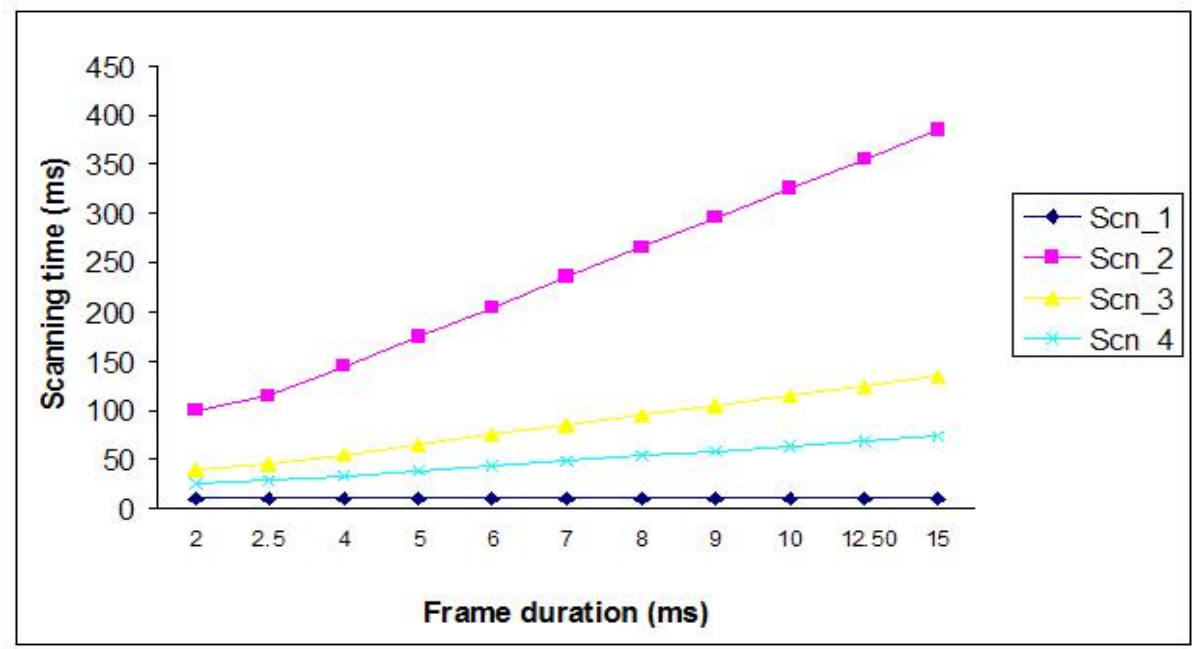

Fig. 6. The effect of frame duration on the scanning time

\section{Conclusions}

This paper analyses handover's scanning process of IEEE802.16m. Since the standard is still under development, the paper assumes similar handover procedure as it is described in IEEE802.16e. But, we are considering the $802.16 \mathrm{~m}$ 's system profiles in our analysis.

The analysis focuses on scanning time and handover interruption time. Based on our analysis, there are some inefficient issues in 802.16e's handover procedure which is critical for $802.16 \mathrm{~m}$. These are redundant scanning and association process of 
neighbour BSs. Additionally, the defined handover strategies also contribute the overhead in handover procedure. The results suggest using the scanning process without association since it has the lowest scanning time. In addition, the handover with the fast ranging and pre-registration is favorable since it has the lowest interruption time.

The IEEE802.16m standard is designed to support high speed mobility therefore the handovers procedures can be expected to very frequently occur. Thus, the scanning process plays an important role in the handover procedure. In our future work, we would like to investigate the single BS scanning and avoiding the inefficient handover by means of mobility prediction.

Acknowledgement. This work has been performed in the framework of FP7 project ROCKET ICT-215282, which is funded by European Commission. The research work was further supported by grant of Czech Ministry of Education, Youth and Sports No. MSM6840770014.

\section{References}

1. Rec. ITU-R M.1645: Framework and overall objectives of the future development of IMT2000 and systems beyond IMT-2000 (January 2003)

2. IEEE P802.16e/D12, Air Interface for Fixed and Mobile Broadband Wireless Access Systems: Amendment for Physical and Medium Access Control Layers for Combined Fixed and Mobile Operation in Licensed Bands, New York (December 2005)

3. Wang, L., Liu, F., Ji, Y.: Perfomance Analysis of Fast Handover Schemes in IEEE802.16e Broadband Wireless Networks. In: Asia Pacific Advanced Network (2007)

4. Choi, S.: Fast handover scheme for real-time downlink services in IEEE 802.16e BWA systems. In: Proceeding of IEEE 61st Vehicular Technology Conference, vol. 3, pp. 2028-2032 (2005)

5. Jiao, W., Jiang, P., Ma, Y.: Fast Handover Scheme for Real-Time Applications in Mobile WiMAX. In: Proceeding of International Conference on Communication (ICC 2007), Glasgow, Scotland (June 2007)

6. IEEE 802.16m-07/002r4, IEEE 802.16m System Requirements (October 2007)

7. Lee, H., Wong, W.C., Sydir, J., Johnsson, K., Yang, S., Lee, M.: MS MAC handover Procedure in an MR Network - Network Topology Advertisement, Proposal paper on IEEE 802.16j, CTP 06/218 (November 2006)

8. IEEE 802.16m-08/003r7, IEEE 802.16m System Description Document (February 2009)

9. Lee, D.H., Kyamakya, K., Umondi, J.P.: Fast Handover Algorithm for IEEE802.16e Broadband Wireless Access System. In: Proceeding of the 1st International Symposium on Wireless Pervasive Computing (2006) 